\title{
A Tunable Approach to Visualize BRCA1 Assemblies in Hereditary Breast Cancer
}

\author{
Carly E. Winton ${ }^{1,2}$, Brian L. Gilmore ${ }^{1}$, Andrew C. Demmert ${ }^{1}$, Zhi Sheng ${ }^{1}$, and Deborah F. Kelly ${ }^{1,2}$ \\ ${ }^{1}$ Virginia Tech Carilion School of Medicine and Research Institute, Roanoke, VA 24016, USA. \\ ${ }^{2}$ School of Biomedical Engineering and Science, Virginia Tech, Blacksburg, VA 24061, USA.
}

Invasive breast cancer remains a leading killer of women in the U.S. today (www.cancer.org). Germline mutations in the breast cancer susceptibility protein (BRCA1) are highly correlated with hereditary forms of the disease, accounting for $\sim 25 \%$ of all diagnosed cases [1]. Currently, patients with BRCA1 mutations have poor clinical outcomes due to the highly aggressive nature of the tumors and high recurrence rates following conventional therapies [2]. These challenges create a major impetus to elucidate the molecular underpinnings of the disease in an effort to improve treatment options.

Under normal cellular conditions, BRCA1 acts as a global genomic surveyor through its diverse roles in DNA repair and RNA synthesis. In the nucleus, BRCA1 can interact with its binding partner BARD1 (BRCA1 Associated RING domain protein) as well as with RNA Polymerase II (RNAP II) [3]. To date, there is no information for the precise manner in which BRCA1 associates with larger protein assemblies, as conventional purification techniques have not yielded complexes suitable for structural analysis. To address this issue, we have developed a new tunable microchip approach that allows us to recover BRCA1-associated protein complexes from the nuclear material of patient-derived breast cancer cells for the first time (Figure 1). This alternative strategy to standard biochemical purification techniques preserves native protein-protein interactions for subsequent biochemical and cryo-Electron Microscopy (EM) analysis.

Our new strategy utilizes Silicon Nitride (SiN) microchips coated with Nickel-nitrilotriacetic acid (NiNTA) lipid films that can be easily decorated with a variety of adaptor molecules including His-tagged protein A and a myriad of antibodies, thus creating a tunable system. We employed this system to capture and visualize active BRCA1-transcriptional complexes from the nuclear extracts of hereditary breast cancer cells. We collected images of the tethered BRCA1 assemblies under low-dose condition using TEM. We processed the images using the PARTICLE software package, and the RELION package [4] was then used to calculate a composite 3D density map of the complex. Employing a combination of antibody-labeling and molecular modeling techniques, we could uniquely place the RNAP II core structure into the density map along with the BRCA1-BARD1 RING domains and BRCA1 C-terminal (BRCT) domain. We could further position a short strand of DNA and K63-linked ubiquitin moieties within the map in areas with additional minor density (Figure 2). Complementary biochemical experiments were used to confirm our structural findings and to reveal the first glimpse of BRCA1 assemblies in the context of human cancer.

\section{References:}

[1] Miki Y., Swensen J., Shattuck-Eidens D., Futreal P. A., Harshman K., Tavtigian S., et al., Science. 266 (1994), pp.66-71.

[2] Charafe-Jauffret E., Ginestier C., Iovino F., Wicinski J., Cervera N., Finetti P., et al., Cancer Res. 69 (2009), pp. 1302-13.

[3] Friedman L. S., Ostermeyer E. A., Lynch E. D., Szabo C. I., Anderson L. A., Dowd P., et al., Cancer Res. 54 (1994), pp. 6374-82.

[4] S.H. Scheres., J. Mol. Biol. 415 (2012), pp. 406-418. 


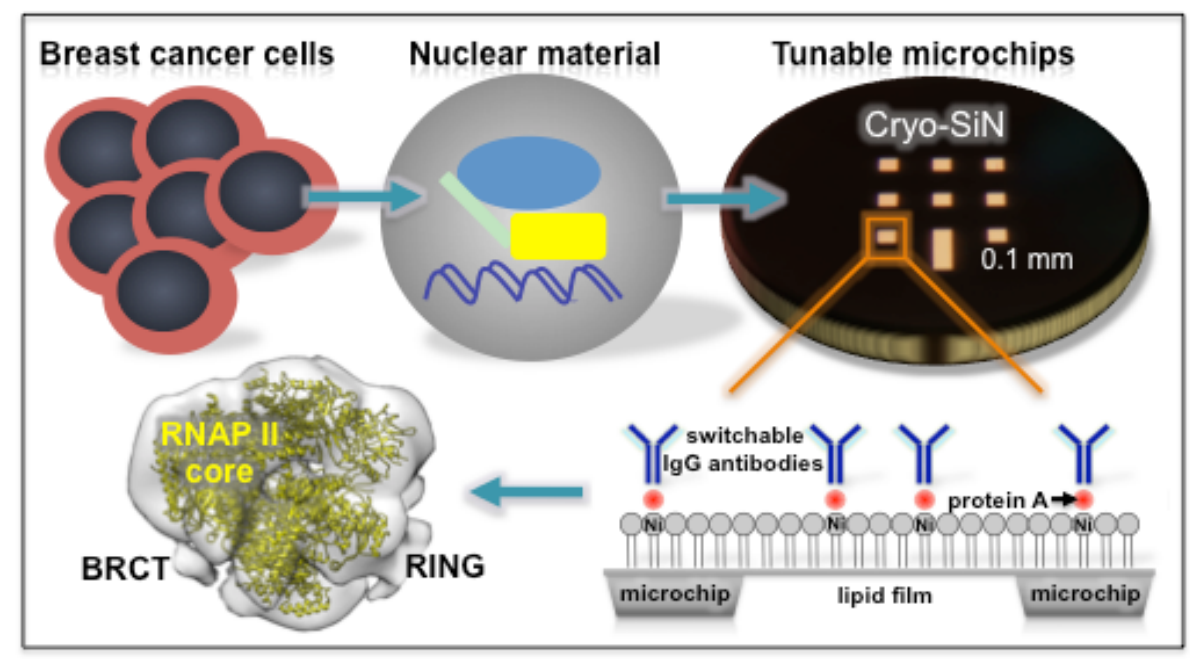

Figure 1. Patient-derived breast cancer cells were lysed and the nuclear material was collected and enriched in RNAP II, BRCA1, and BARD1. The enriched nuclear material was then loaded onto tunable microchips decorated with Protein A and switchable IgG antibodies. The RNAP II core structure was uniquely placed in the resulting EM density map and regions of BRCA1 RING and BRCT domains were identified using antibody-labeling techniques.

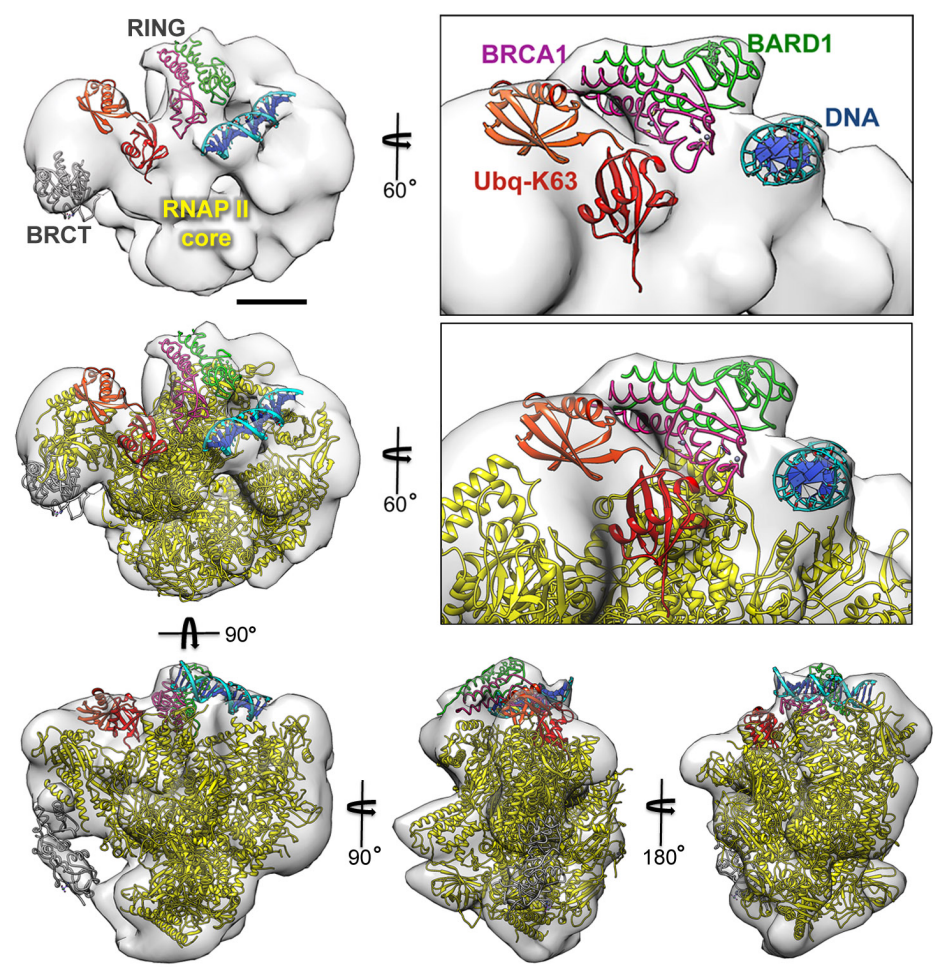

Figure 2. The RNAP II core (yellow) fit uniquely in the density map while additional major densities were attributed to the BRCA1-BARD1 RING domains (magenta, green) and BRCT domain (gray). A short strand of DNA (blue) and K63-linked ubiquitins (red, orange) were placed within regions of minor unoccupied densities. We are testing this model using patient-derived cells harboring BRCA1 mutations. 